\title{
Modelling Drying Process in Paper Manufacturings
}

\author{
Adérito Araújo ${ }^{1} \dagger$, Filomena Teodoro $^{2}$, Gonçalo Pena ${ }^{1}$, Joã quim \\ Correia $^{3}$, Jo Ão Penedones ${ }^{4}$, Jorge Santos ${ }^{5}$, José Augusto Ferreira ${ }^{1}$, Luísa \\ Morgado $^{2}$ LS Silvia Barbeiro ${ }^{1}$ and Stella Abreu ${ }^{5}$ \\ ${ }^{1}$ University of Coimbra, Portugal \\ ${ }^{2}$ CEMAT, University of Lisbon, Portugal \\ ${ }^{3}$ University of Evora, Portugal \\ ${ }^{4}$ EPFL, Switzerland \\ ${ }^{5}$ University of Porto, Portugal \\ (Communicated to MIIR on 15 April 2021)
}

Study Group: ESGI 86. 7-11 May 2012, Porto, Portugal.

Communicated by: Tim Myers

Industrial Partner: Euroresinas - Industrias Quimicas, S. A.

Presenter: Fernando Guimarães and Pedro Mena

Team Members: Adérito Araújo, Coimbra; Filomena Teodoro, Lisbon; Gonçalo Pena, Coimbra; Joãquim Correia, Evora; João Penedones, Lausanne; Jorge Santos, Porto; José Augusto Ferreira, Coimbra; Luísa Morgado, Lisbon; Sılvia Barbeiro, Coimbra; Stella Abreu, Porto.

Industrial Sector: Manufacturing

Tools: Finite elements, Fluid dynamics

Key Words: Paper manufacturing

MSC2020 Codes: 65,76

\section{Summary}

EURORESINAS produces different types of resin-impregnated paper at their factories. After the paper goes through the resin bath, it undergoes a drying process which is a fundamental step in the process. Although the temperature is controlled by a heating system, the actual values inside the chain of dryers are only measured at a few points away from where the paper is. The company would like to be able to model the temperature profile inside the dryers to better understand problems such as dusting and sticking, as well as the optimization of different variables related to bathing and drying times and energy consumption.

$\dagger$ Corresponding Author: alma@mat.uc.pt 


\section{Introduction}

Impregnated papers with resins are widely used in decorative surface finishing panels for indoor and outdoor engineering applications. In the production process, the paper, also referred to as web, is, at a first stage, impregnated with a certain amount of resin. This amount is called the resin content or resin pick up. After going through a resin bath, the paper is lead through a drying process. The solvent, which is normally water, is removed by the drying process, producing a residual moisture on the final film, called the volatile content.

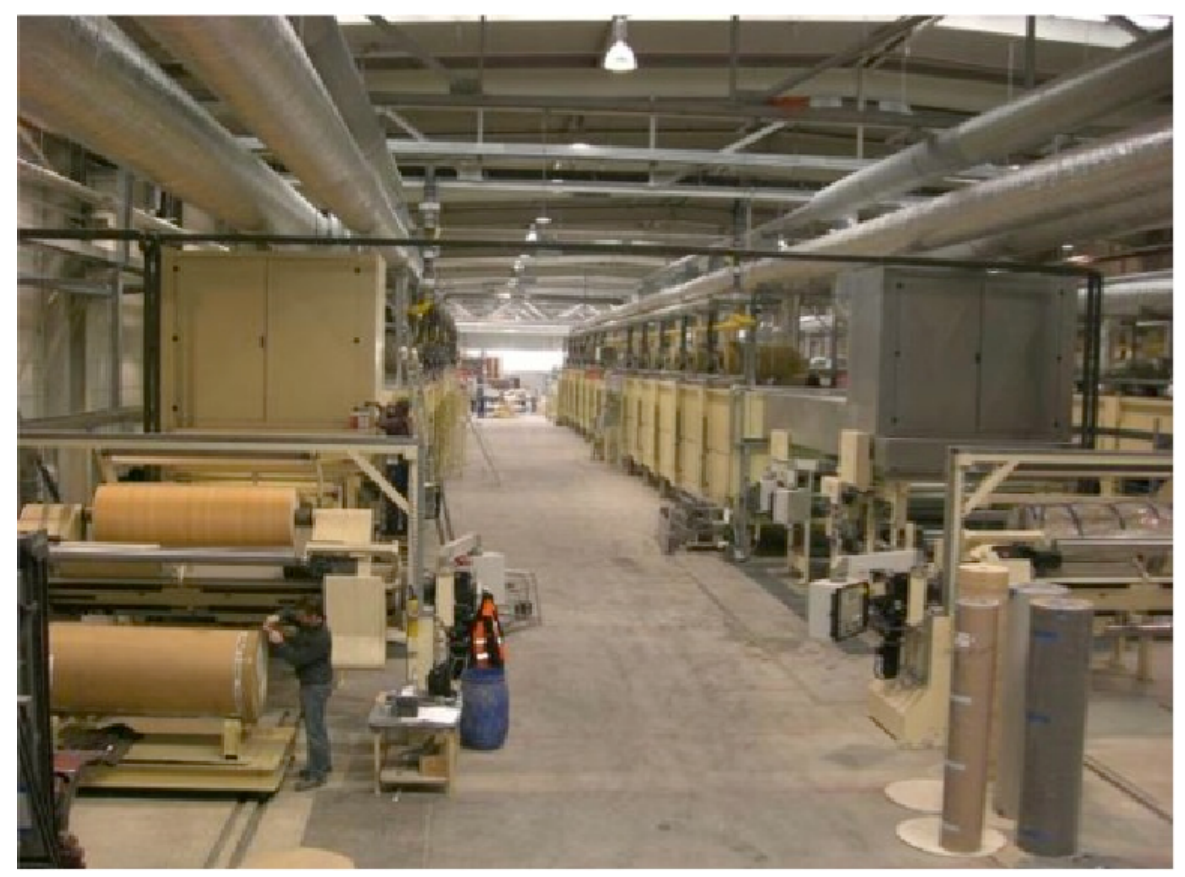

Figure 1: Production line of one of Euroresinas' factories. 
In order to minimize the production costs and maximize the quality of the final product, it is of great importance to understand the relationships between manufacturing conditions and paper quality parameters.

It is known that relevant paper quality parameters, like thickness, temperature and moisture among other, are significantly influenced by manufacturing variables, like the velocity of the film and the number, length and temperature of the dryers (see, for example, Cepitis [3], Buikis [2], A. Asheim [1], Cyr [4], Figueiredo [5], Frydrich [6] and Reardon [7], among others).

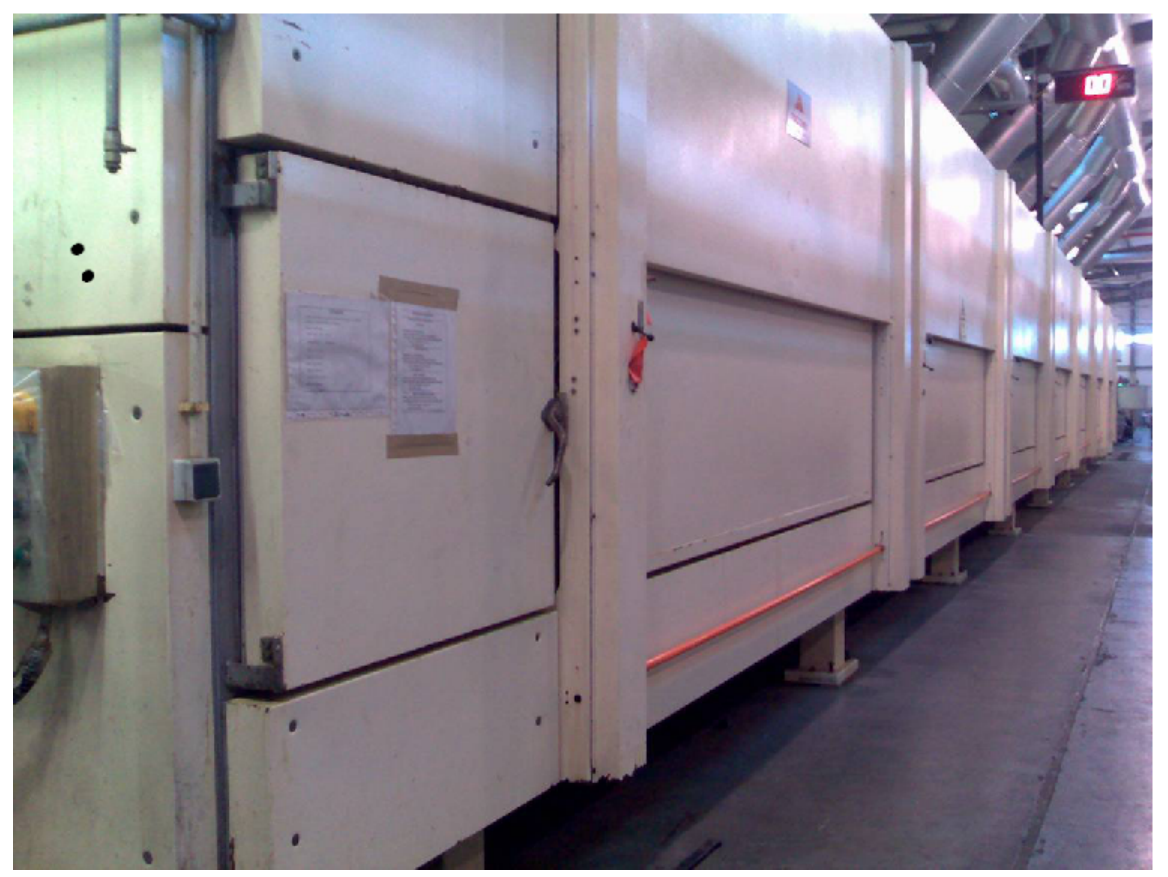

Figure 2: Dryers for the phenolic production line at Sines, Portugal.

EURORESINAS (see figures 1 and 2) produces different types of resin-impregnated paper at their factories: phenolic, melaminic and urea.

While in the production line using phenolic resin, the film goes along 7 consecutive dryers (Figure 3a), in the production line using melamine resin, it passes through $5+7$ dryers (Figure $3 \mathrm{~b}$ ). In both cases, each dryer has a certain number of heaters with a fixed temperature.

Although the temperature is controlled by a heating system, the actual values inside the chain of dryers are only measured at a few points away from where the paper is actually flowing. Also, the air inside the dryers is recirculated and the humidity removed through proper vents (see Figure 4 for a schematic detail of a typical production line).

The challenge proposed to the $86^{\text {th }}$ ESGI is to develop a mathematical model that can predict the temperature of the web along the dryers length 


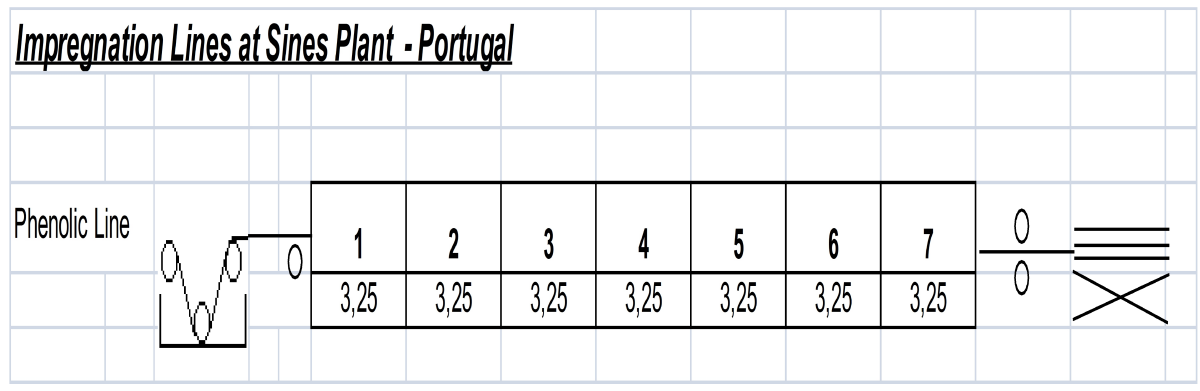

(a) Phenolic production line at Sines, Portugal

\section{Impregnation Lines at Kaisersesch Plant - Germany}

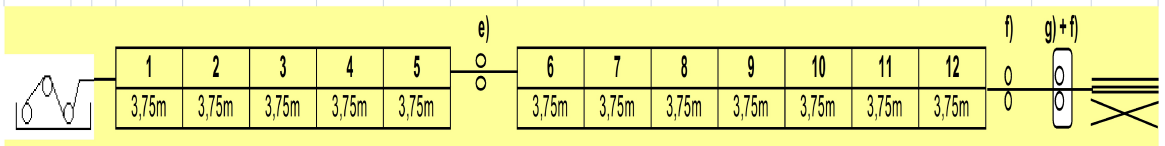

(b) Melamine production line at Kaisersesch, Germany

Figure 3: Two production lines of Euroresinas.

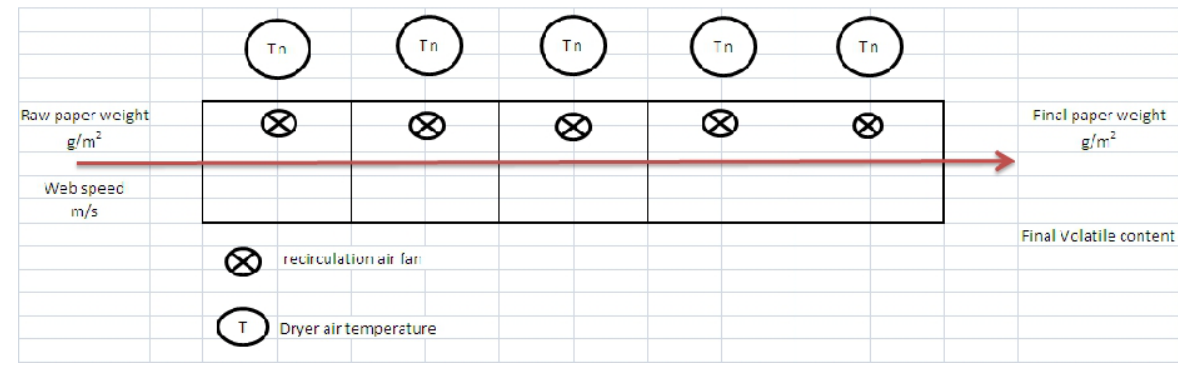

Figure 4: Scheme of the dryers in a production line.

as a function of the relevant variables, such as: raw paper weight, final paper weight, residual moisture (volatile content), web speed, air dryer's temperature, number and length of dryers and air fan recirculation speed.

The final goal, which is out of the scope of this work, is to better understand problems such as dusting and sticking, as well as the optimization of different variables related to bathing and drying times and energy consumption.

This report is organized as follows: in Section 2 we derive the mathematical model. We start by describing the simplified geometrical representation of the production line. The model we consider in this domain consists in a system of partial differential equations (PDEs) to describe the temperature inside the dryers and a system of ordinary differential equations (ODEs) to describe the the temperature and the amount of water of the paper. We 
integrated the model numerically using finite elements for the PDE and the implicit Euler method for the ODEs system as presented in Section 3. In Section 4 we carry out some numerical experiments in order to observe the behavior of the system and the influence of different parameters in the temperature profiles. Finally, in Section 5, we give general conclusions and we point out future directions to proceed with this work.

\section{Mathematical modeling}

Pursuing the objectives, which consist on improving the operating efficiencies of existing machines by analysing the fundamentals of the physics of the drying processes, we will construct a mathematical model to simulate the process. The process that takes place inside the drying machine is particularly complex. Our option was to build a mathematical model to represent the dynamic equilibrium of the system in two phases, which are described in the sub-sections 2.2 and 2.3. In Section 2.3 we will consider a mathematical model predict the temperature and the percentage of water in the paper film in the process drying taking into account the several variables mentioned at the last section. The evolution of the variables is described by a system of ODEs, one for each primary variable (temperature and partial vapor pressure of water of the mixture). The temperature of the hot air in the dryers must be previously computed, since that information is crucial in the description of the ODEs system. This is done by solving a system of PDEs describing the temperature inside of the dryers. This two-dimensional model will be presented in Section 2.2. In the next subsection we define the geometrical domain.

\subsection{Geometrical representation of the production line}

The production line consists in a series of aligned dryers of hexahedral shape, with air recirculation holes on the surface (figures 2 and 4). Inside each dryer there are fans to make the air flow as well as heaters to increase the air temperature. The paper moves along these dryers, at a constant speed, floating on air, from the entrance to the dryers, until the end of the line. To simplify the modeling of the problem at hand, we consider a simplified geometry to represent the production line.

We start by describing the domain which will be considered for modeling the thermal and drying phenomena inside the dryers and in the paper.

We assume that the dryers are modeled by a two dimensional geometry, neglecting the depth variable. Let $L=3.75 \mathrm{~m}$ and $h=4 \mathrm{~m}$ denote, respectively, the length and height of each dryer. We denote by $\Omega_{i}$ the domain occupied by the i-th dryer in the production line, and by $\Omega$ the union of all dryers. Figure 5 represents the geometry for the particular case of 2 dryers. We provide a brief explanation on the other parts of the boundary depicted 


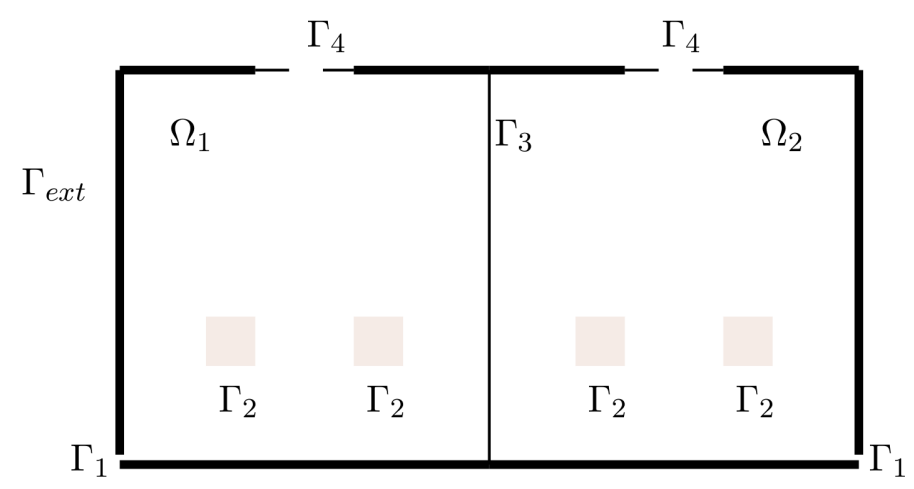

Figure 5: Schematic representation of the domain $\Omega$ and boundaries $\Gamma_{1}, \Gamma_{2}$, $\Gamma_{3}, \Gamma_{4}$ for the case of two dryers.

in Figure 5:

- the boundary $\Gamma_{3}$ is the wall (walls if there are more than just two dryers) that separate each two consecutive dryers;

- $\Gamma_{2}$ represents the heaters in each dryer, modeled as squares of length $l_{\text {heaters }}=50 \mathrm{~cm}$ and at fixed distance from the sides of each dryer (distance to the sides, $d_{\text {heaters,side }}=1 \mathrm{~m}$, and distance to the bottom of $\Omega, d_{\text {heaters,bottom }}=1 \mathrm{~m}$ );

- $\Gamma_{4}$ models the heat exhaustion vents in the surface of the dryers as line segments, centered at the middle of the top boundary of each dryer, and with length $l_{\text {vents }}=1 \mathrm{~m}$;

- $\Gamma_{1}$ is the part of boundary of $\Omega$ in which the paper goes into the dryers;

- and $\Gamma_{e x t}=\partial \Omega \backslash\left(\Gamma_{4} \cup \Gamma_{1} \cup \Gamma_{2}\right)$.

It is important to notice that from a geometrical point of view, $\Gamma_{1}, \Gamma_{2}$ and $\Gamma_{4}$ are subsets of the boundary of $\Omega$. In fact, $\Omega$ is the rectangular region shown in Figure 5 with the rectangular holes determined by $\Gamma_{2}$.

We assume that the paper moves along and over the bottom edge of the domain $\Omega$, at constant velocity $v$ (units $\mathrm{m} / \mathrm{s}$ ).

\subsection{The hot air model}

We propose a mathematical model to describe the steady-state behavior of the temperature inside the dryer machines, through the following PDEs

$$
-\nabla \cdot\left(K \nabla T_{d, i}\right)+V \cdot \nabla T_{d, i}=0, \text { in } \Omega_{i}, i=1, \ldots, n_{\text {dryers }}
$$


and with the coupling conditions

$$
\begin{aligned}
T_{d, i} & =T_{d, i+1}, \text { on } \overline{\Omega_{i}} \cap \bar{\Omega}_{i+1} \\
\nabla T_{d, i} \cdot \eta_{i} & =-\nabla T_{d, i+1} \cdot \eta_{i+1}, \text { on } \overline{\Omega_{i}} \cap \bar{\Omega}_{i+1}
\end{aligned}
$$

for $i=1, \ldots, n_{\text {dryers }}-1$, where $T_{d, i}$ is the temperature in $\Omega_{i}, K$ is the air thermal conductivity, $V$ is the heaters air velocity and $\eta_{i}$ the external unit normal associated with $\partial \Omega_{i}$. Note that the conditions (2)-(3) enforce the continuity of the temperature and temperature flux across consecutive dryers.

Remark. In order to simulate air flow inside the dryers, we considered a (constant in time) velocity $V$ defined all over $\Omega$. A more complete description of the hot air flow problem should include a velocity $V$ obtained as the solution of the Navier-Stokes equations coupled with the temperature equation, as opposed to the approach now considered which considers a prescribed space dependent function for velocity.

Remark. For $i=1, \ldots, n_{\text {dryers }}-1$, we denote $\Gamma_{3}^{i}=\overline{\Omega_{i}} \cap \bar{\Omega}_{i+1}$ and notice that

$$
\Gamma_{3}=\bigcup_{i=1}^{n_{\text {dryers }-1}} \Gamma_{3}^{i} .
$$

In an analogous fashion, let $\Gamma_{j}^{i}=\Gamma_{j} \cap \bar{\Omega}_{i}$, for $j=1,2,4$ and $i=1, \ldots, n_{\text {dryers }}$ and $\Gamma_{\text {ext }}^{i}=\Gamma_{\text {ext }} \cap \bar{\Omega}_{i}$ for $i=1, \ldots, n_{\text {dryers }}$.

For $i=1$ and $i=n_{\text {dryers }}$, the following boundary conditions (BC) are imposed to the unknowns $T_{d, i}$ :

$$
T_{d, i}=T_{a i r}, \quad \text { on } \Gamma_{1}^{i},
$$

where $T_{a i r}$ is the ambience air temperature.

For all the dryers, i.e., for $i=1 \ldots, n_{\text {dryers }}$, we also set

$$
\begin{aligned}
& T_{d, i}=T_{\text {heat }}, \quad \text { on } \Gamma_{2}^{i}, \\
& \nabla T_{d, i} \cdot \eta_{i}=\epsilon\left(T_{d, i}-T_{a i r}\right), \quad \text { on } \Gamma_{4}^{i} \text {, } \\
& \nabla T_{d, i} \cdot \eta_{i}=0, \quad \text { on } \Gamma_{e x t}^{i},
\end{aligned}
$$

where $T_{\text {heat }}$ is the temperature of the heaters and $\epsilon$ is the extraction rate (which is related with air fan recirculation speed).

The variational formulation for the coupled problem (1)-(7) reads as: 
for $i=1, \ldots, n_{\text {dryers }}$, find $T_{d, i} \in H_{\Sigma}^{1}\left(\Omega_{i}\right)$ such that

$$
\begin{aligned}
& K \int_{\Omega_{i}} \nabla T_{d, i} \cdot \nabla v_{i} d x+\int_{\Omega_{i}} V \cdot \nabla T_{d, i} v_{i} d x \\
&+K \int_{\Gamma_{3}^{i}}^{i} \nabla T_{d, i} \cdot \eta_{i} v_{i} d s \\
&-K \int_{\Gamma_{3}^{i-1}} \nabla T_{d, i-1} \cdot \eta_{i-1} v_{i} d s \\
&+\epsilon K \int_{\Gamma_{4}^{i}}\left(T_{d, i}-T_{a i r}\right) v_{i} d s=0, \forall v_{i} \in H_{\Sigma, 0}^{1}\left(\Omega_{i}\right)
\end{aligned}
$$

and

$$
\int_{\Gamma_{3}^{i}} T_{d, i} \mu_{i} d s=\int_{\Gamma_{3}^{i}} T_{d, i+1} \mu_{i} d s, \forall \mu_{i} \in L^{2}\left(\Gamma_{3}^{i}\right)
$$

where

$$
H_{\Sigma}^{1}\left(\Omega_{i}\right)=\left\{v \in H^{1}\left(\Omega_{i}\right): v_{\mid \Gamma_{1}^{i}}=T_{\text {air }} \text { and } v_{\mid \Gamma_{2}^{i}}=T_{\text {heat }}\right\}
$$

and

$$
H_{\Sigma, 0}^{1}\left(\Omega_{i}\right)=\left\{v \in H^{1}\left(\Omega_{i}\right): v_{\mid \Gamma_{1}^{i}}=0 \text { and } v_{\mid \Gamma_{2}^{i}}=0\right\} .
$$

\subsection{The paper model}

Assuming that the temperature profile at the bottom of the dryers are known, we are able to use this information to construct a model to describe the drying process in the paper film.

We shall look for a time-independent solution that describes the system working in a stationary state. The basic unknowns are the temperature profile $\tilde{T}$ and water density profile $\tilde{\rho}$ of the paper along the dryer. We will choose $x=0$ at the entrance and $x=L$ at the exit of the dryer. The paper moves inside the dryer at constant velocity $v$ (units $\mathrm{m} / \mathrm{s}$ ). Therefore, the spatial profiles $\tilde{T}, \tilde{\rho}$ are equivalent to the time evolution of the temperature $T$ and water density $\rho$ of a piece of paper that passes through the dryer. In other words,

$$
\tilde{T}(x)=T(x / v) \quad \tilde{\rho}(x)=\rho(x / v), \quad t=x / v,
$$

and the time evolution starts at $t=0$ and ends at $T_{\text {end }}=L / v$. We will now write equations that determine this time evolution on $I=\left(0, T_{\text {end }}\right]$.

The first equation controls the temperature increase of the paper:

$$
c(t) \dot{T}(t)=j_{\text {ext }}(t)+\lambda \dot{\rho}(t), t \in I
$$

where $c$ is the specific heat of the paper per unit area (units $J /\left(K m^{2}\right)$ ) defined by

$$
c(t)=c_{H_{2} O} \rho(t)+c_{r} \rho_{r}+c_{p} \rho_{p}, t \in I
$$


with $c_{\mathrm{H}_{2} \mathrm{O}}, c_{r}, c_{p}$ specific heat per unit mass (units $J /(K \mathrm{~kg})$ ) of water, resin and dry paper, respectively. The variables $\rho, \rho_{r}$ and $\rho_{p}$ stand for mass per unit area (units $\mathrm{kg} / \mathrm{m}^{2}$ ) of the same substances. On the right hand side of equation (13), we have two heat fluxes (units $W / m^{2}$ ). The first one corresponds to heat flow from the hot air in the dryer. Its value is controlled by the temperature in the dryer and also by the geometry. One naive estimate is to write

$$
j_{e x t}=\sigma_{a i r}\left(T_{e x t}-T\right)
$$

where $\sigma_{\text {air }}$ (units $W /\left(K m^{2}\right)$ ) is a transference rate which depends on the thermal conductivity of the air and on the properties of the resin and $T_{\text {ext }}$ is the hot air temperature at the paper surface. This estimate could be improved using the full model of heat flow in the dryer to determine the constant of proportionality between $j_{\text {ext }}$ and $T_{\text {ext }}-T$. We remark that the temperature $T_{\text {ext }}$ can be obtained by a convenient interpolation operator applied to the function $T_{d}$ over the bottom edge of $\Omega$.

The second heat flow describes the heat loss due to water evaporation. The parameter $\lambda$ is the water latent heat per unit mass (units $J / k g$ ).

The evaporation rate is controlled by the Hertz-Knudsen equation

$$
\dot{\rho}(t)=-\alpha \sqrt{\frac{m_{H_{2} O}}{2 \pi k_{B} T(t)}}\left(P(t)-P_{e x t}(t)\right), t \in I
$$

where $m_{H_{2} O}$ is the mass of a molecule of water, $\alpha$ is a parameter to slow down the evaporation and $k_{B}$ is the Boltzmann constant. $P$ is the partial vapor pressure of water of the mixture water, resin and paper. This can be estimated (using Raoult's law) by

$$
P(t)=\frac{\rho(t)}{\rho(t)+\rho_{p}+\rho_{r}} P_{v a p}(T(t)), t \in I
$$

where

$$
P_{v a p}(T)=P_{0} \exp \left(1-\frac{T_{0}}{T}\right)
$$

is the vapour pressure of water at a given temperature. The parameters $P_{0}$ and $T_{0}$ are known experimentally. Finally, $P_{\text {ext }}(t)$ is the partial pressure of water in the hot air inside the dryer. This depends on the humidity and temperature of the air via

$$
P_{\text {ext }}=x_{H_{2} O} P_{\text {vap }}\left(T_{\text {ext }}\right)
$$

where $x_{\mathrm{H}_{2} \mathrm{O}}$ is the molecular fraction of water in the air (ratio between the number of water molecules per unit volume and the total number of molecules per unit volume of air). In practice, we did something much simpler and set this pressure to a constant

$$
P_{\text {ext }} \equiv P_{\text {vap }}\left(T_{0}\right)
$$


where $T_{0}$ is the initial temperature of the paper.

This system of equations can be integrated for any chosen $T_{e x t}$ and initial conditions

$$
T(0)=T_{0}, \quad \rho(0)=\rho_{0} .
$$

\section{Numerical algorithm}

A numerical algorithm to solve the differential equations presented in Section 2 can be set in a straightforward way:

1st step: Solve the coupled problem (1)-(7) numerically;

2nd step: Extract the temperature profile, $T_{e x t}$, at the boundary $\Gamma_{e x t}$, on the bottom of the dryers;

3rd step: Compute the temperature profile, $T$, and water density profile, $\rho$, of the paper web, by solving numerically (13), (16), (21).

The computation of a numerical solution of (1)-(7) shall be addressed by the finite element method, while the time integration of the system of ODEs from step three, shall be done by implicit Euler's method.

In the next sections, we detail and explain the numerical procedures used in each step.

\subsection{Numerical method for the hot air equation}

We start this section by introducing some notations. Let $h>0$ denote a positive real. For $i=1, \ldots, n_{\text {dryers }}, \mathcal{T}_{i, h}$ denotes a collection of triangulations of $\Omega_{i}$, respectively, such that $\mathcal{T}_{i, h}$ and $\mathcal{T}_{i+1, h}$ are conform on $\Gamma_{3}^{i}$, for $i=$ $1, \ldots, n_{\text {dryers }}-1$. Let $\mathcal{T}_{h}$ denote a triangulation obtained as the union of all

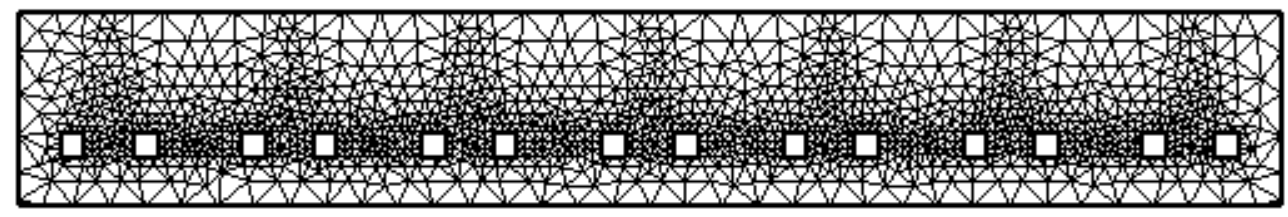

Figure 6: Triangular mesh in the domain for the case of 7 dryers.

$\mathcal{T}_{i, h}$, for $i=1, \ldots, n_{\text {dryers }}$ (see Figure 6 for the case of 7 dryers). Let $\mathbb{P}\left(\mathcal{T}_{i, h}\right)$ denote the space of piecewise linear polynomials built upon the triangulation $\mathcal{T}_{i, h}$. 
The weak formulation for the finite element discretization of (8)-(9) reads as: for $i=1, \ldots, n_{\text {dryers }}$, find $T_{d, i, h} \in \mathbb{P}\left(\mathcal{T}_{i, h}\right) \cap H_{\Sigma}^{1}\left(\Omega_{i}\right)$ such that

$$
\begin{aligned}
& K \int_{\Omega_{i}} \nabla T_{d, i, h} \cdot \nabla v_{i, h} d x+\int_{\Omega_{i}} V \cdot \nabla T_{d, i, h} v_{i, h} d x \\
&+K \int_{\Gamma_{3}^{i}} \nabla T_{d, i, h} \cdot \eta_{i} v_{i, h} d s-K \int_{\Gamma_{3}^{i-1}} \nabla T_{d, i-1, h} \cdot \eta_{i-1} v_{i, h} d s \\
&+\epsilon K \int_{\Gamma_{4}^{i}}\left(T_{d, i, h}-T_{a i r}\right) v_{i, h} d s=0, \\
& \forall v_{i, h} \in \mathbb{P}\left(\mathcal{T}_{i, h}\right) \cap H_{\Sigma, 0}^{1}\left(\Omega_{i}\right)
\end{aligned}
$$

and

$$
\int_{\Gamma_{3}^{i}} T_{d, i, h} \mu_{i, h} d s=\int_{\Gamma_{3}^{i}} T_{d, i+1, h} \mu_{i, h} d s, \forall \mu_{i, h} \in \mathbb{P}\left(\mathcal{T}_{i, h} \cap \mathcal{T}_{i+1, h}\right) .
$$

The set of equations (23) enforces, at the discrete level, the continuity of the temperature functions across the interface $\Gamma_{3}^{i}$. We can combine all the functions $T_{d, i, h}$, while assuming the continuity just explained, into a function $T_{d, h} \in \mathbb{P}\left(\mathcal{T}_{h}\right)$. In fact, if

$$
T_{d, h \mid \Omega_{i}}=T_{d, i, h}
$$

then the variational formulation $(22)-(23)$ can be recast in terms of this global temperature as: find $T_{d, h} \in \mathbb{P}\left(\mathcal{T}_{h}\right) \cap H_{\Sigma}^{1}(\Omega)$ such that

$$
\begin{aligned}
K \int_{\Omega} \nabla T_{d, h} \cdot \nabla & v_{h} d x+\int_{\Omega} V \cdot \nabla T_{d, h} v_{h} d x \\
+\epsilon K \int_{\Gamma_{4}}\left(T_{d, h}-T_{a i r}\right) v_{h} d s & =0, \forall v_{h} \in \mathbb{P}\left(\mathcal{T}_{h}\right) \cap H_{\Sigma, 0}^{1}(\Omega)
\end{aligned}
$$

where $H_{\Sigma}^{1}(\Omega)$ and $H_{\Sigma, 0}^{1}(\Omega)$ are defined as in (10) and (11), respectively, but removing the index $i$ from those definitions.

Without entering into many technical implementation details, the calculation of $T_{d, h}$, solution of of (24), can be performed by solving a linear system, associated with (24).

\subsection{Numerical method for the paper film equations}

For the numerical approximation of (13), (16), (21) we consider the implicit Euler method with a fixed time step for the time discretization. Let $\Delta t>0$ denote the time step and $0=t_{0}<t_{1}<\cdots<t_{n}=T_{\text {end }}$ a uniform partition of $I$ with $t_{i}-t_{i-1}=\Delta t$, for $i=1, \ldots, n$. For $n=1, \ldots, n$, we denote $\rho_{k}$ and $T_{k}$ approximations of $\rho\left(t_{k}\right)$ and $T\left(t_{k}\right)$, respectively. 
The Implicit Euler method applied to the problem (13), (16) and (21) reads as: for $k=0, \ldots, n-1$, find $\rho_{k}$ and $T_{k}$ such that

$$
\begin{aligned}
c_{k+1} \frac{T_{k+1}-T_{k}}{\Delta t}+\lambda \alpha \sqrt{\frac{m_{H_{2} O}}{2 \pi k_{B} T_{k+1}}}\left(P_{k+1}-P_{e x t}\left(t_{k+1}\right)\right) & =0 \\
\rho_{k+1}+\Delta t \alpha \sqrt{\frac{m_{H_{2} O}}{2 \pi k_{B} T_{k+1}}}\left(P_{k+1}-P_{e x t}\left(t_{k+1}\right)\right) & =\rho_{k}
\end{aligned}
$$

where $c_{k+1}$ and $P_{k+1}$ are defined as in (17) and (14), respectively, replacing $\rho\left(t_{k+1}\right)$ by $\rho_{k+1}$ and $T\left(t_{k+1}\right)$ by $T_{k+1}$.

The solution of the nonlinear system (25)-(26) is calculated with a quasiNewton method, defined as the usual Newton's method, but approximating the jacobian of the nonlinear operator with finite differences.

Indeed, if for each $k$, we define

$\mathbf{F}_{k}(T, \rho)=\left[\begin{array}{c}\left(c_{H_{2} O} \rho+c_{r} \rho_{r}+c_{p} \rho_{p}\right) \frac{T-T_{k}}{\Delta t}+\lambda \alpha \sqrt{\frac{m_{H_{2} O} O}{2 \pi k_{B} T}}\left(\frac{\rho}{\rho+\rho_{p}+\rho_{r}} P_{v a p}(T)-P_{e x t}\left(t_{k+1}\right)\right) \\ \rho+\Delta t \alpha \sqrt{\frac{m_{H_{2} O} O}{2 \pi k_{B} T}}\left(\frac{\rho}{\rho+\rho_{p}+\rho_{r}} P_{\text {vap }}(T)-P_{\text {ext }}\left(t_{k+1}\right)\right)\end{array}\right]$

then the quasi-Newton method reads as:

set $T_{k+1,1}=T_{k}$ and $\rho_{k+1,1}=\rho_{k}$ and for $i=1, \ldots$, calculate

$$
\left\{\begin{array}{l}
\mathbf{J}_{k+1, i+1} \delta_{k+1, i+1}=-\mathbf{F}_{k}\left(T_{k+1, i}, \rho_{k+1, i}\right) \\
\left(T_{k+1, i+1}, \rho_{k+1, i+1}\right)=\delta_{k+1, i+1}+\left(T_{k+1, i}, \rho_{k+1, i}\right)
\end{array}\right.
$$

until $\left\|\delta_{k+1, i+1}\right\|<10^{-10}$. When the prescribed tolerance is achieved, say, for an index $i^{*}$, we set $T_{k+1}=T_{k+1, i^{*}+1}$ and $\rho_{k+1}=\rho_{k+1, i^{*}+1}$.

\section{Numerical simulations}

In our numerical simulations we have used the program Matlab and the free software FreeFem ++ (www.freefem.org).

Some numerical simulations were carried out assuming that the impregnated paper moves, at a constant velocity $v$, along $5+7$ consecutive dryers, each one with a fixed air temperature at each heater $T_{\text {heat }}$ (see Figure $3 \mathrm{~b}$ ). We consider the ambience air temperature $T_{a i r}=25^{\circ} \mathrm{C}(=298 \mathrm{~K})$. The initial values $T_{0}=T_{\text {air }}$ and $\rho_{0}=0.7$ were considered. In the re-empregnation phase that occurs in the gap between the first 5 dryers and the last 7 dryers, we consider that the percentage of humidity on the paper is equal to $\rho_{0}$.

In Table 1 we present the data for the numerical results in Figure 7 (cases (a), (b) and (c)). We consider changes in air temperature $T_{\text {heat }}$ in the heaters and the extraction coefficient $c_{e x t}$ in each dryer. Changes in the number of heaters, in the distance between them and in the distance between the heaters and the web can also be simulated. 


\begin{tabular}{|c|ccccc|ccccccc|c|}
\hline & \multicolumn{10}{|c|}{ Heater's temperature in each dryer } & $c_{\text {ext }}$ \\
\hline (a) & 100 & 110 & 131 & 110 & 100 & 145 & 151 & 155 & 150 & 141 & 130 & 120 & 0.005 \\
\hline (b) & 100 & 110 & 131 & 110 & 100 & 145 & 151 & 155 & 150 & 141 & 130 & 120 & 0.001 \\
\hline (c) & 100 & 110 & 131 & 110 & 100 & 120 & 130 & 140 & 150 & 140 & 130 & 120 & 0.005 \\
\hline
\end{tabular}

Table 1: Temperature $T_{\text {heat }}$ (in degrees Celsius) and extraction coefficient $c_{\text {ext }}$ constant in at all extraction vents.

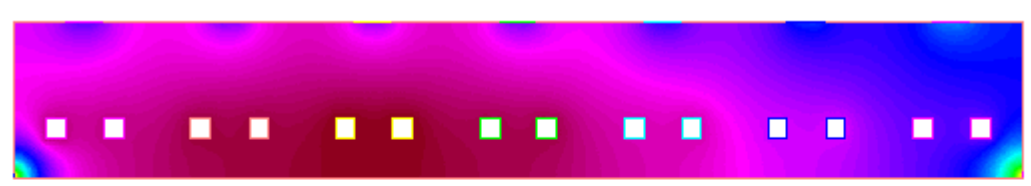

(a)
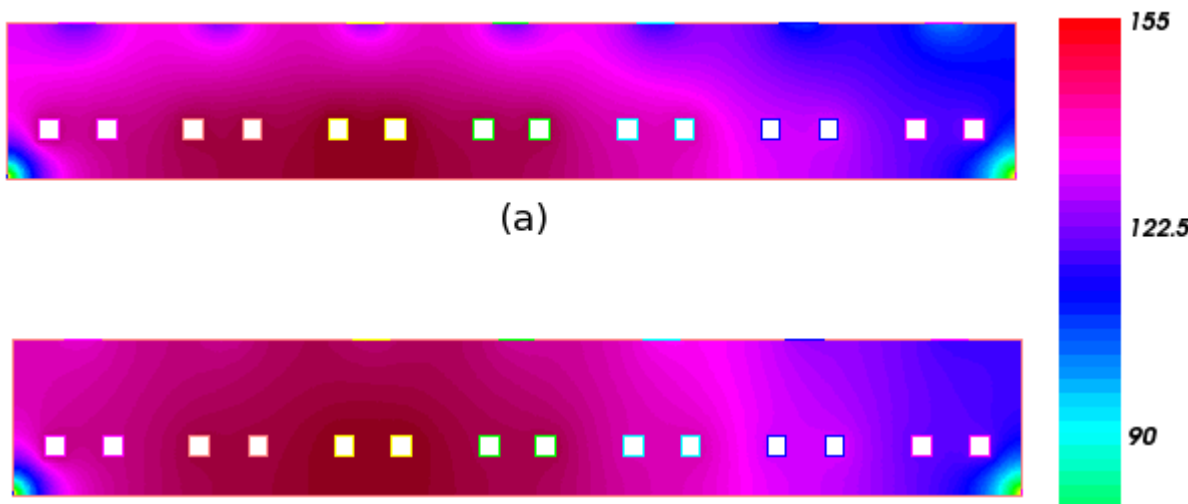

(b)

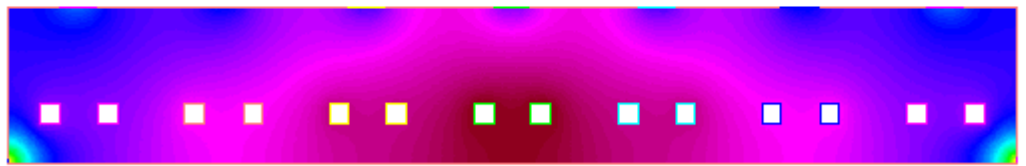

(c)

Figure 7: Temperature (in degrees Celsius) along the last 7 dryers using the data in Table 1.

As we see in Figure 7, changes in the parameters $T_{\text {heat }}$ and $c_{\text {ext }}$ clearly influences the temperature inside the dryers and may be used to control the temperature on the paper surface.

The temperature $T(t)$ profile along the production line $(5+7$ dryers $)$ on the paper surface for the data in Table 1 is presented in Figure 8. As we can observe, the temperature on the paper surface is not the same as in the heaters but, as expected, is strongly related. The influence of the parameter $c_{\text {ext }}$, for this example, is not so high as expected. From (a) to (b) we decrease its value five times and the temperature profile on the paper surface is almost the same.

The profiles of the temperature $T(t)$ and humidity $\rho(t)$ for different values of the velocity $v$ are presented in Figure 9 and $\sigma_{\text {air }}=3170 \mathrm{~W} /\left(\mathrm{Km}^{2}\right)$. As it can be observed, the velocity of the film highly influences the tem- 


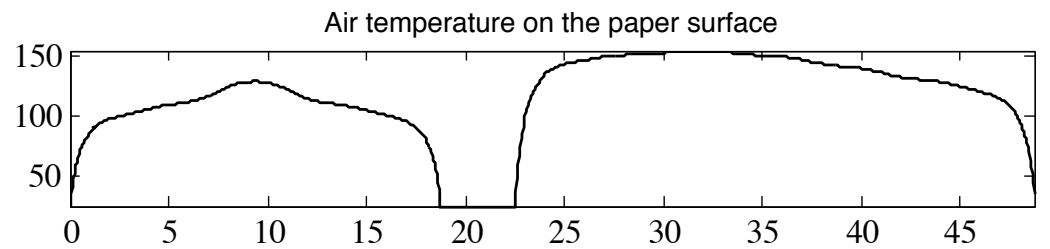

(a)

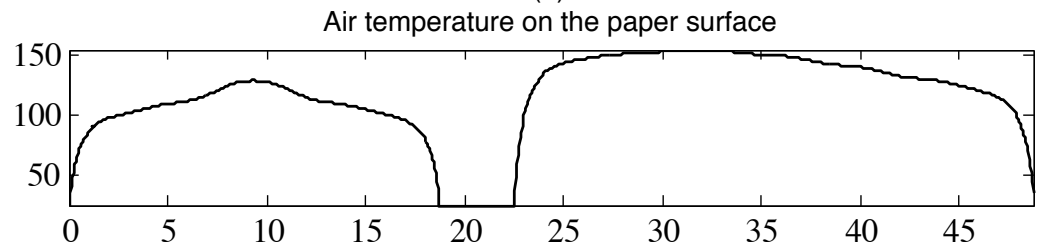

(b)

Air temperature on the paper surface

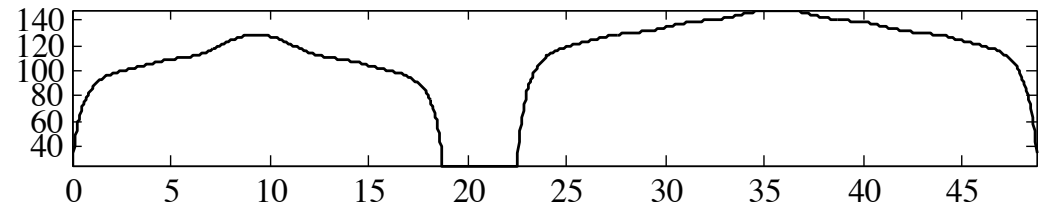

(c)

Figure 8: Temperature profile (in degrees Celsius) along the production line $(5+7$ dryers $)$ on the paper surface using the data in Table 1 .

perature profile. In particular, when the percentage of humidity is beyond a certain level, the temperature rises sharply. This clearly show that the percentage of humidity at the atmosphere (that is taken into account in the parameter $\rho_{0}$ ) have an effect on the final temperature of the paper.

In Figure 10, we fix the velocity of the film on $v=2.5 \mathrm{~m} / \mathrm{s}$ (which is the usual used velocity of the film in the phenolic line) and vary the parameter $\sigma_{\text {air }}$. We can conclude from a strong dependence of the temperature profiles on the properties of the resin.

\section{Conclusions and recommendations}

The objective of the present project was to understand the process of drying resin-impregnated papers in a industrial heating system. In order to do so, we have set up a mathematical model where we have considered the main physical aspects of the process based on fundamental laws of physics .

The resulting model consists in a system PDEs to describe the temperature inside the dryers and a system of ODEs to describe the state variables of the paper, namely the temperature and the amount of water. We integrated the model numerically using finite elements for the PDEs system and the implicit Euler method for the ODEs system. The implementation of the EDPs-ODEs system was done in FreeFem ++ and Matlab. We used the 

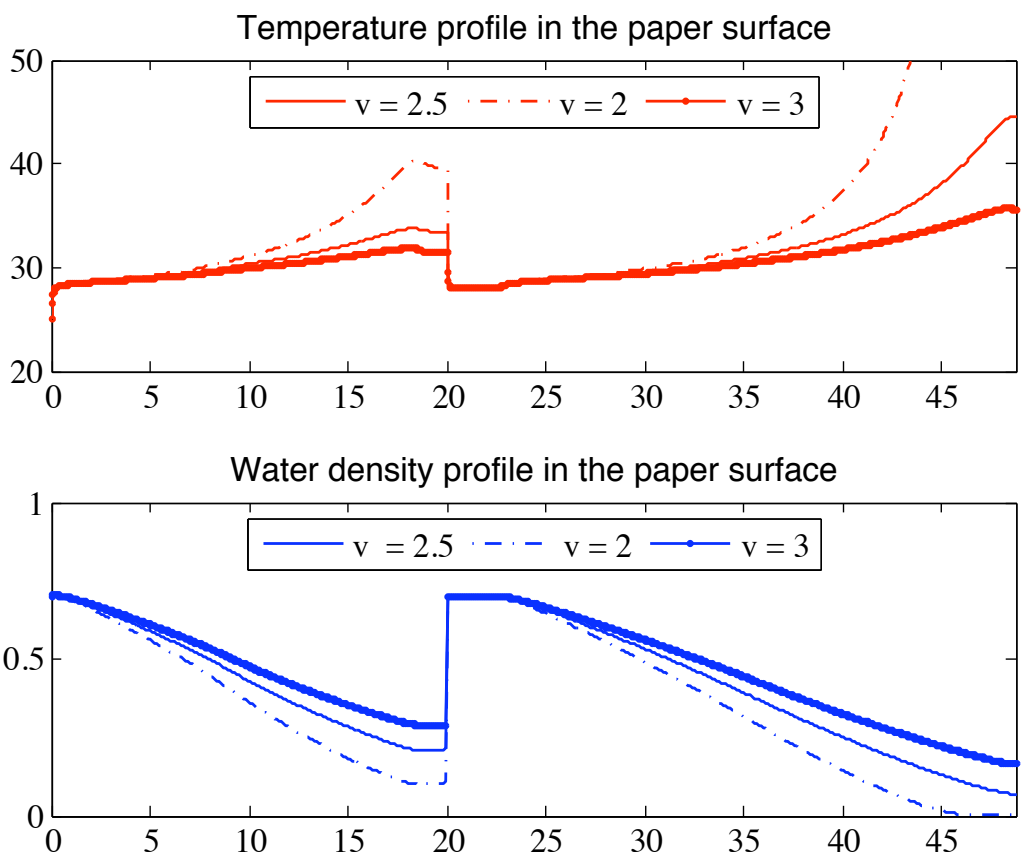

Figure 9: Temperature (in degrees Celsius) and humidity profiles along the production line $(5+7$ dryers $)$ on the paper surface for different velocities of the web, using the data in example (a) in Table 1 and $\sigma_{\text {air }}=3170 \mathrm{~W} /\left(\mathrm{Km}^{2}\right)$.

model to obtain some conclusions about the behavior of the system and the influence of different parameters in the temperature profiles. In the results we observe that air temperature inside the dryers and room temperature have a big impact.

Even though the EDPs-ODEs model yield good qualitative results, it must be validated using experimental data. This is a crucial step that can be carried out in a close collaboration with the industrial partner to be done in the future. We believe that the model here described can provide some useful hints with interest for the optimization of production process.

\section{References}

[1] A. Asheim, A. Lutowska, O. Martinez-Alvarado, V. Sachers, A. Sinwel, M. Aguareles, M. Pellicer, Modelling the ironing process in industrial laundry, Proceedings of the European Student Workshop on Mathematical Modelling in Industry, 19th ECMI Modelling Week, Barcelona (2006) 2-20. 

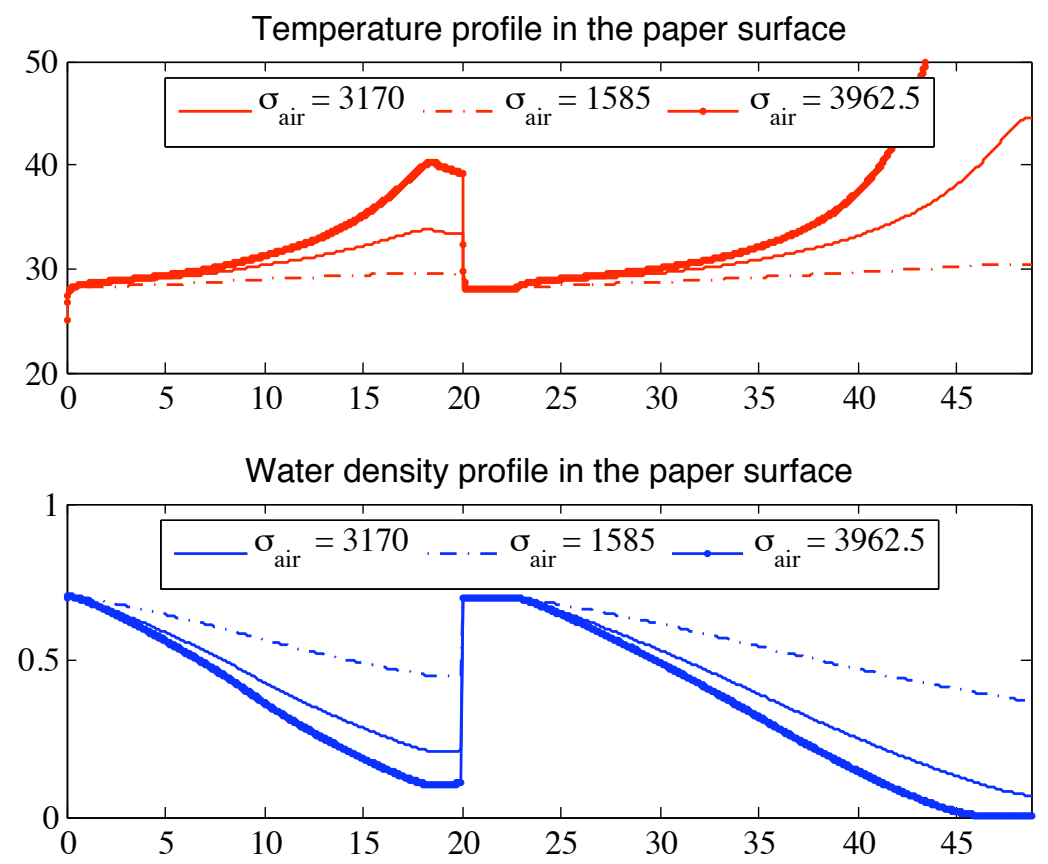

Figure 10: Temperature (in degrees Celsius) and humidity profiles along the production line ( $5+7$ dryers) on the paper surface for different values of $\sigma_{a i r}$, using the data in example (a) in Table 1 and $v=2.5 \mathrm{~m} / \mathrm{s}$.

[2] A. Buikis, J. Cepitis, H. Kalis, A. Reinfields, A. Ancitis, A. Salmins. Mathematical models of Papermaking, Non linear Analysis, 6 (1) (2001) 9-19.

[3] J. Cepitis. A mathematical model of paper drying, Mathematical Modelling and Analysis, 5 (1) (2000) 26-31.

[4] P.-L. Cyr, B. Riedl, X.-M. Wang, L. Groom, S. M. Shaler, UreaMelamine-Formaldehyde Resin Diffusion into Medium Density Fiberboard Wood Fibers, J. Adhes. Sci. Technol. , 20 (8) (2006) 787-801.

[5] A. B. Figueiredo, D. V. Evtuguin, J. Monteiro, E. F. Cardoso, P. C. Mena, P. Cruz. Structure Property Relaionsships of Kraft Papers: Implication on Impregnation with Phenol- Formaldehyde Resin, Industrial and Engineering Chemistry Research 50 (5) (2011) 2883-2890.

[6] D. Frydrich and P. Ralek, Solution of Coupled Heat and Moisture Diffusion with Sorption for Textiles, Proceedings of ALGORITMY 2005, $53-63$. 
[7] S. A. Reardon, M. R. Davis and P. E. Doe. Computational modelling of paper drying machines. Tappi Journal 83 (9) (2000). 